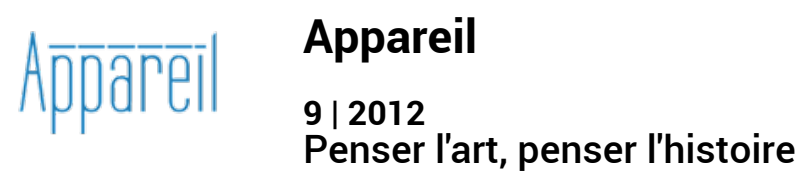

\title{
Art et temps
}

Les catégories historiques à l'épreuve de l'art préhistorique

\section{Audrey Rieber}

\section{CpenEdition}

\section{Journals}

Édition électronique

URL : http://journals.openedition.org/appareil/1442

DOI : 10.4000/appareil. 1442

ISSN : 2101-0714

Éditeur

MSH Paris Nord

\section{Référence électronique}

Audrey Rieber, «Art et temps », Appareil [En ligne], 9 | 2012, mis en ligne le 05 juillet 2012, consulté le 30 juillet 2020. URL : http://journals.openedition.org/appareil/1442 ; DOI : https://doi.org/10.4000/ appareil. 1442

Ce document a été généré automatiquement le 30 juillet 2020.

\section{(c) $(1) \odot$}

Appareil est mis à disposition selon les termes de la Licence Creative Commons Attribution - Pas d'Utilisation Commerciale - Pas de Modification 4.0 International. 


\section{Art et temps}

Les catégories historiques à l'épreuve de l'art préhistorique

\section{Audrey Rieber}

\section{Introduction}

1 Nombreux sont les historiens de l'art à proposer une conception globale de l'évolution artistique, de ses débuts jusqu'à nos jours. Quelle que soit la diversité de leurs conceptions, cycliques, organiques, téléologiques, un point les rassemble: la méconnaissance des œuvres préhistoriques. Or n'est-il pas surprenant qu'une histoire de l'art qui réfléchit ses origines ignore ses tout premiers commencements? Plusieurs raisons éclairent ce paradoxe. La première est d'ordre disciplinaire et tient au fait que le paléolithique est l'objet de la science préhistorique et non de l'histoire de l'art. À cette raison institutionnelle s'en ajoute une autre, scientifique ou épistémologique. Si en dépit de la fascination qu'exercent les œuvres préhistoriques sur le plus large public, elles ne sont quasiment pas envisagées par l'histoire de l'art (et guère par la Bildwissenschaft), c'est parce que leur appréhension requiert une imposante somme de connaissances scientifiques qu'on ne peut se targuer de posséder facilement. Une appréhension un peu sérieuse de cet objet requiert en effet des connaissances en paléontologie, anthropologie, géologie, histoire des migrations, des civilisations, des techniques, etc. Inversement, les archéologues et préhistoriens n'envisagent que rarement leurs objets sous l'angle artistique. Les récents livres de Jean Clottes, L'art des cavernes (2008) et Pourquoi l'art préhistorique? (2011), constituent une exception. En faisant le constat que les productions préhistoriques ne sont pas envisagées par l'histoire de l'art, je n'entends ni proposer à celle-ci un objet encore inédit ni lui suggérer de reconsidérer la question de l'origine de l'art ou de la signification de l'art paléolithique, mais voir dans quelle mesure la prise en compte de ces œuvres permet d'interroger une certaine façon d'écrire l'histoire (de l'art) ${ }^{1}$. Est-il par exemple encore possible de concevoir l'histoire de l'art comme celle du perfectionnement de l'imitation après la découverte de la grotte de Chauvet et sa comparaison avec celle de Lascaux (figures 1 et 2)? 
Figure 1

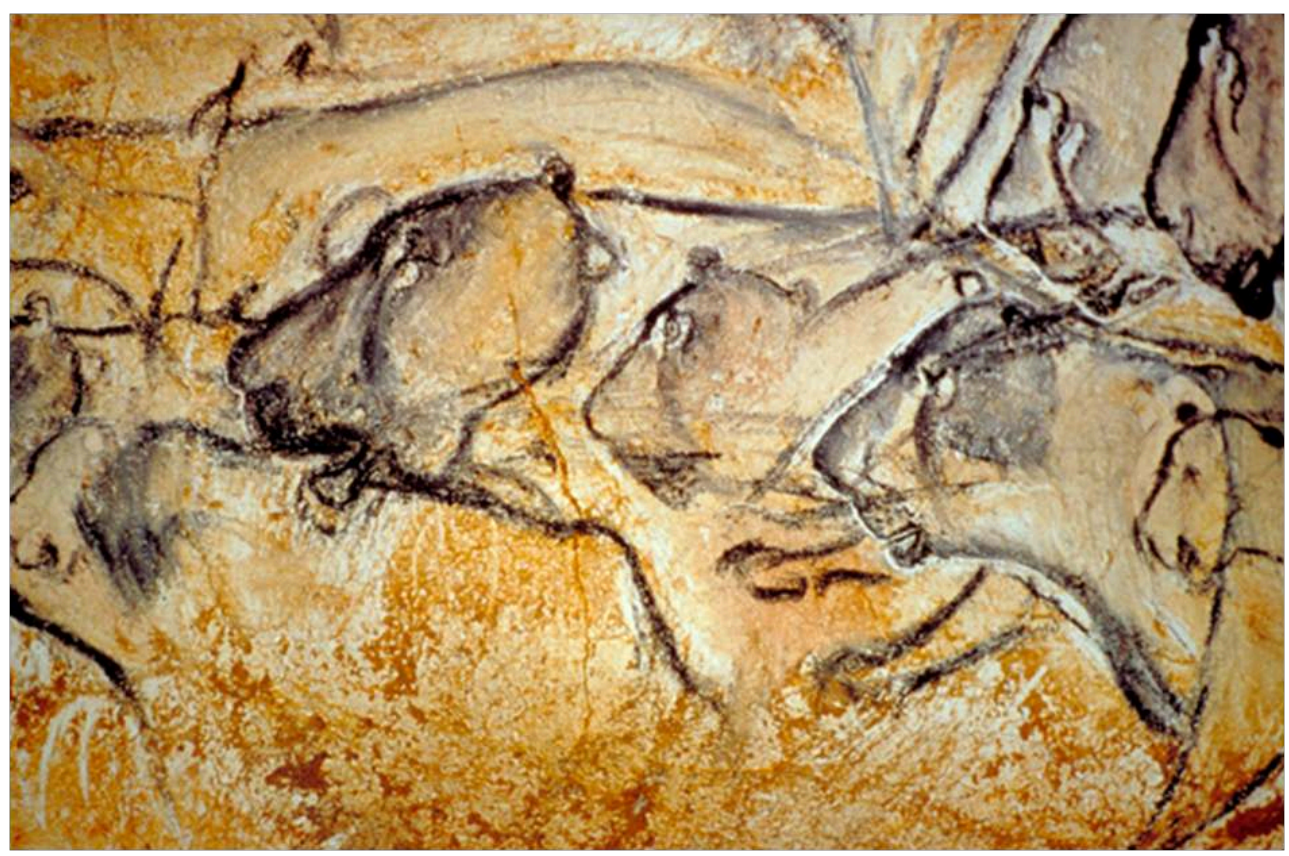

Grotte Chauvet, Vallon-Pont-d'Arc, Ardèche. Panneau des lions (détail). Dessin sur roche. Longueur : environ $400 \mathrm{~cm}$. Aurignacien.

Figure 2

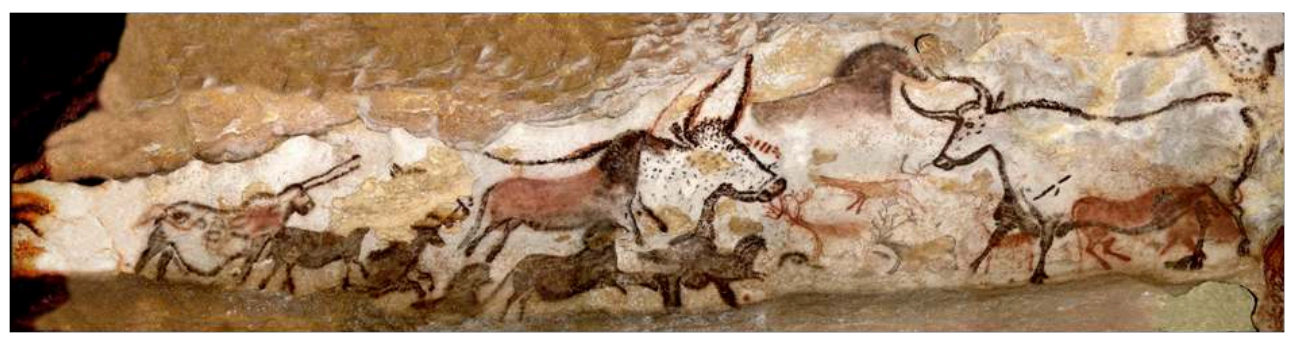

Grotte de Lascaux, Montignac, Dordogne. Salle des taureaux. Peinture sur roche. Longueur de la licorne : $235 \mathrm{~cm}$. Solutréen / Magdalénien ancien.

2 On pourrait bien sûr objecter à notre questionnement que l'histoire de l'art ne peut, par définition, tenir compte des objets préhistoriques. Mais l'idée d'un avant l'histoire est peu claire et l'on sait que la notion de préhistoire est controversée, sa datation engageant des critères tant chronologiques qu'ethnologiques et philosophiques. Néanmoins, si l'exclusion de principe des productions préhistoriques du champ de l'histoire de l'art est injustifiée, ce serait faire à beaucoup d'historiens un procès anachronique que de leur reprocher de les passer sous silence. D'une part, on trouve bien chez Vasari, l'un des fondateurs de la discipline, le souci de faire débuter l'histoire de l'art aux origines, c'est-à-dire à la Genèse, où Dieu, en formant l'homme, "fit paraître ainsi, à travers cette superbe création, la première idée de la sculpture et de la peinture $^{2}$ ", d'autre part et surtout, la notion même de préhistoire n'apparaît que tardivement. Elle se constitue comme discipline au cours du XIX ${ }^{e}$ siècle, de la fin duquel datent les premières découvertes: Altamira (Espagne) est découvert en 1879, Chabot (Gard) en 1878/1889, La Mouthe (Dordogne) en 1895, Pair-Non-Pair (Gironde) en 1896, 
Marsoulas (Haute-Garonne) en 1897 et Les Combarelles (Dordogne) en 1901³. Les découvertes se sont ensuite multipliées. Même si certains sites étaient connus auparavant, ils n'étaient pas pensables en termes de préhistoire ${ }^{4}$. Tenant compte du temps des découvertes et de l'histoire de la science préhistorique, je n'évoquerai donc que des historiens de l'art du $\mathrm{xx}^{\mathrm{e}}$ siècle.

On pourrait encore opposer à la tentative d'examiner les schémas temporels de l'histoire de l'art à l'aune des productions préhistoriques que celles-ci ne relèvent pas de l'art. À ceux qui considèrent qu'elles n'appartiennent pas à l'art parce qu'en plus d'être faibles techniquement, elles ne sont pas issues d'une intention esthétique, on peut rappeler, premièrement, la complexité technique de certaines créations et, deuxièmement, l'argument de Riegl affirmant l'existence d'un Kunstwollen préhistorique. Dans sa polémique contre Gottfried Semper et (surtout) ses successeurs, qui font dériver les propriétés formelles de l'art de sa fonction, de sa technique et de son matériau, Riegl évoque un manche de poignard préhistorique sculpté dans un os de renne (figure 3) et déclare :

L'incitation n'est pas du tout venue de la technique, mais de la volonté explicite de faire de l'art. On voulait recréer dans un matériau inerte l'image d'un être de la nature, et l'on a su inventer la technique nécessaire à cette fin. Pour une bonne préhension, il n'était pas nécessaire qu'un manche de poignard portât la figuration complète d'un renne. Sans doute un instinct artistique immanent, présent en l'homme et qui, dès son émergence, a lutté pour s'imposer, a conduit [...] au façonnage de cette poignée d'os en forme de renne ${ }^{5}$.

Figure 3

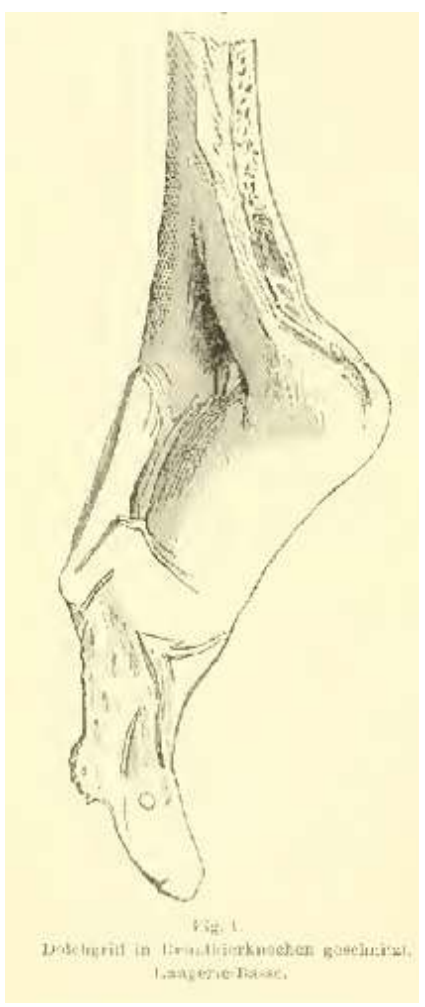

Manche de poignard sculpté dans un os de renne (Laugerie-Basse, Les Eyzies-de-Tayac, Dordogne), musée d'Archéologie nationale, Saint-Germain-en-Laye, in A. Riegl, Stilfragen. Grundlegungen zu einer Geschichte der Ornamentik, Berlin, Georg Siemens Verlag, 1893, p. 16. 
(L'affirmation d'une volonté d'art est aussi dirigée contre William Henry Goodyear qui rend compte des motifs ornementaux par leur symbolique solaire.) On peut douter que l'élaboration des peintures rupestres résulte d'une volonté « explicite » de faire de l'art. À en croire les recherches les plus récentes, leur élaboration relevait de rituels chamaniques ${ }^{6}$. En outre, concernant ce poignard, on peut imaginer qu'une fonction sociale entrait aussi en jeu. Plusieurs aspects de la réflexion de Riegl peuvent néanmoins être retenus : l'affirmation d'une compétence technique des préhistoriques, l'existence de préoccupations formelles et, enfin, l'idée qu'une même pulsion créatrice nous relie à ces hommes. D'autres arguments encore pourraient être proposés pour justifier l'appartenance de ces productions à l'art (et nous aurons l'occasion d'en évoquer certains), mais le point sur lequel j'aimerais insister concerne moins la nature de l'art (ou la définition que l'historien de l'art s'en fait) que celle de la temporalité. Quelle sorte d'histoire permet-elle de faire une place à l'art préhistorique ? Mieux : de quelle conception du temps devons-nous nous défaire pour le penser, non plus avant ou hors, mais avec le reste des productions artistiques?

1.

5 Plus intéressant encore que de constater que les historiens de l'art consacrent peu de réflexions à la préhistoire est d'analyser les difficultés auxquelles conduisent les tentatives de la penser. Exemplaires de ce point de vue sont les réflexions d'E. H. Gombrich et d'André Malraux. L'Histoire de l'art (1950) de Gombrich, ouvrage de vulgarisation mais qui n'en témoigne pas moins des fondements de sa pensée, s'ouvre sur les «étranges débuts» de l'art: celui «de la préhistoire, des primitifs et de l'Amérique avant sa découverte ${ }^{7}$ ». La mise en rapport de productions géographiquement et temporellement aussi distinctes a de quoi surprendre; elle constitue une sorte de doxa que l'on rencontre dès qu'on aborde la question du "primitif». Entreprendre sa généalogie et sa critique dépasse le cadre du présent article; signalons simplement que, s'il est souvent purement idéologique, "l'usage prudent de l'analogie ethnographique " peut posséder une portée méthodologique ${ }^{8}$. Gombrich avance des arguments assez classiques pour justifier l'appartenance des productions préhistoriques à l'art. Qu'elles aient été liées à des pratiques magiques (la représentation des animaux étant censée faciliter leur capture ${ }^{9}$ ) ne les exclut pas du champ de l'art, puisqu'à ce compte, très peu d'œuvres que nous considérons comme artistiques le seraient. Par ailleurs, qu'elles soient issues d'une production codifiée ne suffit pas davantage à les disqualifier, puisque, d'une part, les contraintes fonctionnelles et formelles ne sont pas propres à l'art primitif et que, de l'autre, cellesci laissent toujours à l'artiste la possibilité de manifester ses préférences, son goût et sa technique. Gombrich souligne également la maîtrise technique parfois étonnante des primitifs. Si certaines de leurs œuvres présentent une apparence curieuse, cela n'est dû ni à leur maladresse ni à une incapacité technique, mais à une conception du monde spécifique. En affirmant, enfin, que l'histoire de l'art n'est pas celle de progrès techniques, mais de conceptions et de besoins changeants, Gombrich, curieusement, ne semble pas loin de défendre la théorie du Kunstwollen de Riegl qu'il critique par ailleurs durement ${ }^{10}$.

6 Mais les analyses de ce premier chapitre font état de plusieurs réserves concernant l'intégration à l'histoire de l'art des peintures découvertes à Lascaux, Altamira et Font 
de Gaume. D'abord, et bien qu'il s'en défende, Gombrich considère bien les primitifs comme des primitifs : «Ils ne sont pas plus simples que nous, mais tout de même plus proches des débuts de l'humanité11 ». Cette proximité avec les origines suggère que les primitifs sont à un stade encore embryonnaire du développement, et la comparaison avec l'enfant devient inévitable. "Certains nègres d'Afrique sont tout aussi peu conscients de la différence entre image et réalité que des enfants ${ }^{12}$ ». L'incapacité à produire un art mimétique caractérise également l'art préhistorique, l'art tribal, l'art folklorique, les dessins d'enfants et ceux des personnes non instruites (untutored art) ${ }^{13}$ ! Gombrich, ensuite, tend à minimiser la dimension artistique des productions qu'il appelle primitives. Il ne parle d'" art " à leur propos qu'entre guillemets, non, dit-il, parce qu'elles seraient privées de beauté, mais parce que «nous ne devons assurément pas nous imaginer qu'elles ont été créées pour être de purs bijoux et parures ${ }^{14} »$. Or n'a t-il pas expliqué plus haut que le fait que ces œuvres possédaient une fonction ne les exclut pas du champ de l'art? Enfin, et c'est le point décisif pour notre propos, Gombrich les écarte de l'histoire qu'il définit comme la transmission d'une tradition. Le critère de l'avènement de l'écriture n'intervient pas, Gombrich soulignant au contraire la parenté qui existe dans ces cultures précoces (in diesen frühen Kulturen) entre l'image et l'écriture ${ }^{15}$. L'exclusion de l'art préhistorique de l'histoire de l'art s'opère au début du chapitre $2:$ «Un art pour l'éternité. Égypte, Mésopotamie, Crète » :

Certes, il y a de l'art partout dans le monde, mais la véritable histoire de l'art ne commence pourtant pas dans les grottes du Sud de la France ou chez les Indiens. Aucun chemin ne conduit de ces débuts curieux et mystérieux à l'art de notre temps ${ }^{16}$.

7 L'argument ne repose ni sur l'affirmation d'une norme esthétique ni sur une définition de l'art auxquelles ces productions ne correspondraient pas, mais sur un argument généalogique, à savoir l'absence de filiation entre ces œuvres et les nôtres, «l'histoire propre de la tradition artistique » commençant en Égypte.

Il existe vraiment une tradition intacte du maître à l'élève, de l'élève à l'imitateur admiratif qui relie toute œuvre visuelle qui est faite aujourd'hui, tout édifice, qui est construit aujourd'hui, oui, n'importe quelle affiche que nous voyons, à des maîtres qui se démenaient il y a 5000 ans sous le soleil égyptien. Nous allons voir que les artistes grecs allaient en apprentissage chez les Égyptiens et que nous tous, que nous soyons modernes ou non, sommes les élèves des Grecs ${ }^{17}$.

L'idée d'une continuité ininterrompue entre l'Égypte et nous explique pourquoi il faut commencer par les anciens Égyptiens lorsqu'on parle de provenance (Herkommen) en art. C'est de là «que vient l'art». L'argument est discutable, en fait et en droit. Gombrich écrit en réalité une histoire de l'art méditerranéo-centrée, dont la haute culture égyptienne marquerait les nobles débuts. Or, non seulement cette focalisation est loin d'être évidente, mais on se demande pourquoi et comment une rupture radicale entre les civilisations préhistorique et égyptienne s'est produite. D'une part, rappelle Clottes, l'art préhistorique ne s'arrête pas avec la fin des temps glaciaires et n'est pas propre à l'Europe ${ }^{18}$. D'autre part, la culture égyptienne n'est pas survenue ex nihilo. L'idée d'un vacuum historique entre la préhistoire et nous relève d'une reconstruction (idéologique) et, avec sa thèse d'une solution de continuité entre la préhistoire et l'Égypte, Gombrich n'est pas loin de prétendre dater le Déluge. Mais si l'idée d'une rupture de tradition est peu crédible, celle d'une évolution linéaire et continue de l'art égyptien au nôtre ne l'est pas davantage. À quoi s'ajoute qu'au sein de la préhistoire déjà, les techniques de représentation ne peuvent être ordonnées linéairement. Dès les époques les plus anciennes (Chauvet), la paroi pouvait être préparée par raclage 
préalable, les contours des animaux peints détourés pour qu'ils ressortent davantage, les pattes ou les corps mis en perspective spatiale, les volumes et le modelé des formes rendus par l'estompe, en étalant la peinture soit à la main, soit avec un tampon. Or non seulement, remarque Clottes, " ces techniques n'allaient pas de soi ", mais " on ne les retrouve [...] que sporadiquement, ailleurs et par la suite ${ }^{19}$ ». Même des historiens qui refusent la notion warburgienne de survivance et travaillent en termes de périodes et de chronologies comme Panofsky défendent l'existence de phénomènes isolés, de ruptures de continuité, de retours en arrière, de tentatives inabouties, de pseudomorphoses (comprises comme l'émergence d'une forme A, analogue, voire identique, par sa morphologie à une forme $\mathrm{B}$, mais dépourvue de tout lien de filiation avec elle) ou encore de recréation spontanée (définie comme la réinvention d'une solution formelle déjà existante mais ignorée par l'artiste $)^{20}$. Remarquable, enfin, est que Gombrich esquisse à propos des primitifs une analyse d'allure warburgienne, mais qui, précisément, n'en a que l'allure. En soutenant que, pour comprendre les étranges origines de l'art, il faut essayer de se mettre dans l'âme des primitifs qui, quelque part en nous, est toujours présente, il semble prêt d'affirmer «l'identité ou plutôt l'indestructibilité de l'homme primitif qui demeure éternellement le même à toutes les époques ${ }^{21} »$. Mais tandis que, chez Warburg, l'approche psychologique des productions culturelles a pour effet de bouleverser les schémas temporels classiques de l'histoire de l'art et de les déchronologiser au profit d'une pensée du Nachleben, elle conduit, chez Gombrich, à exclure de la chronologie historique les productions qui en dévient.

9 La conception continue et linéaire que Gombrich se fait de l'histoire (de l'art) est donc l'un des éléments qui le conduisent à en exclure l'art préhistorique. Ce continuum de l'histoire, il le pense par ailleurs comme orienté vers une fin, à savoir le perfectionnement de la représentation. Or c'est aussi cette structure téléologique de l'histoire que met à mal la prise en compte des productions préhistoriques dont certaines présentent bien des caractères réalistes. Gombrich admet que certaines peintures rupestres produisent un effet étonnamment vivant et naturel, mais c'est pour les priver immédiatement et d'un statut artistique et d'une place dans l'histoire. Elles possédaient essentiellement une utilité magique, dit-il, et il est donc peu vraisemblable qu'elles aient eu une fonction ornementale. Sous prétexte que leur caractère esthétique n'est pas intentionnel, il les rejette du champ artistique, en contradiction avec ses analyses liminaires. Mais il les écarte aussi de l'histoire de la mimésis en soutenant que leur réalisme n'est que partiel. La ressemblance y tient à un seul caractère: on y reconnaît l'ours à sa patte, et non grâce à un traitement stylistique global. À quoi s'ajoute que même lorsqu'elle existe, la reproduction de l'apparence réelle des choses résulte non d'un savoir artistique, mais d'une observation aigue de la nature.

L'impossibilité pour une pensée linéaire, continue et téléologique de l'histoire d'intégrer les productions préhistoriques dans l'histoire est confirmée par l'examen de l'ouvrage (posthume) de Gombrich sur La préférence pour le primitif (2002) (qui peut se traduire aussi bien par un goût pour l'art nègre que par le rejet de l'art officiel des salons). Elle repose, analyse Gombrich, sur une conception organique de l'histoire selon laquelle l'art connaîtrait des phases de développement, de maturité et de déclin. En estimant que les productions de ceux que l'on tient pour sauvages présentent des qualités formelles supérieures aux nôtres, les adeptes du primitif restent dans une perspective du meilleur et du moins bon et ne font que substituer à une normativité classique, académique, une autre, primitiviste. Si Gombrich s'intéresse à cette 
préférence, c'est parce qu'elle l'étonne. Pour lui, qui conçoit l'histoire de l'art comme celle du progrès de la représentation, la prédilection pour des étapes non abouties du rendu mimétique est problématique. Il y voit la tentation de se détourner d'un patrimoine artistique croissant, qui rend la création toujours plus difficile, et la volonté d'améliorer l'expression artistique. Mais pour compréhensible qu'elle soit, cette préférence est disqualifiée : Gombrich ne lui attribue aucune valeur propre (elle est, au sens strict, une réaction), il l'explique par des motifs non artistiques (psychologiques) et refuse aux artistes qui se prétendent primitifs de l'être. (Visant Picasso et Klee, il déclare : «Plus vous préférez le primitif, moins vous pouvez devenir primitif ${ }^{22}$ »). Mais le point qui m'importe n'est pas de critiquer la dimension normative du rejet du primitif par Gombrich - il revendique explicitement l'existence de canons à partir desquels on peut juger la qualité de l'art ${ }^{23}-$, mais d'étudier la dimension temporelle de sa catégorie de primitif qui rend impossible une compréhension historique des productions préhistoriques.

11 Dans le dernier chapitre de son ouvrage, Gombrich s'explique sur l'usage qu'on est en droit de faire du terme de primitif. Il commence par refuser la position évolutionniste propre au XIX siècle qui met les développements culturels en rapport avec la biologie des espèces. Selon elle, l'art primitif est un art élémentaire, plus proche de la vie, bref, enfantin. Il n'est donc pas étonnant, déclare Gombrich, que les premières découvertes de grottes préhistoriques aient été accueillies avec incrédulité, elles qui prouvaient que les hommes de l'âge de pierre étaient de «merveilleux observateurs ${ }^{24}$ ». À l'idée d'une mentalité sauvage que notre culture aurait progressivement dépassée, Gombrich oppose les arguments défendus par Franz Boas dans Primitive Art (1927). La tentative de Boas pour décrire analytiquement les traits fondamentaux de l'art primitif repose sur deux principes : l'identité des processus mentaux dans toutes les races et dans toutes les formes de culture actuelles et l'idée que tout phénomène culturel résulte d'événements historiques. Certes, il dut y avoir une époque où l'équipement mental de l'homme différait du nôtre, mais pour trouver cette autre organisation, il faut remonter aux temps où l'homme commençait à se distinguer des grands singes. Les processus mentaux du primitif ne sont donc ni différents ni inférieurs à ceux de l'homme civilisé ${ }^{25}$. Gombrich en conclut que, pour Boas, les cultures ne doivent pas être rangées selon une échelle ascendante. Or c'est précisément sur l'idée de progrès, que Gombrich identifie d'emblée à celui de l'imitation, que l'historien se sépare de l'anthropologue. Pour Gombrich, ce progrès est d'ordre technique. En raisonnant en termes d'habileté et non d'intention artistique, l'historien tient pour évident que la seule norme valable à laquelle un artiste peut tendre est la mimésis. À Franz Boas qui soutient que l'art tribal ne témoigne nullement d'une technique limitée puisque, lorsque les artistes des tribus du nord-ouest du Pacifique par exemple désiraient la vérité réaliste, ils étaient capables de l'atteindre, Gombrich objecte que ce réalisme ne vaut que pour les œuvres en trois dimensions - une différence, admet-il, qu'il n'a pas faite dans L'art et l'illusion (1960). Certes, il existe des productions primitives réalistes (les têtes dites de remplacement en Égypte, certains vases péruviens, les têtes en bronze d'Ifé - auxquels on pourrait ajouter, pour donner un exemple de primitif magdalénien, les bisons sculptés sur argile retrouvés dans la grotte du Tuc d'Audoubert (figure 4) -, mais il s'agit toujours d'art tridimensionnel. Créer une illusion de réalité en dessinant ou peignant sur une surface plane requiert une «habileté mimétique » (skills of mimesis) qui, loin d'être donnée de tout temps, repose sur un progrès de l'habileté représentative (evolution of representational skill ${ }^{26}$. Et cette habileté est contre-intuitive. Dans le cas de la 
perspective avec point de fuite, par exemple, la représentation mimétique doit surmonter l'obstacle constitué par nos constantes perceptives (qui nous font sousestimer la diminution des carreaux d'un sol rendu en perspective) pour se rapprocher davantage de l'illusion. «L'artiste miméticien doit apprendre non à se concentrer sur la façon dont il voit le monde, mais à ignorer son expérience visuelle en faveur d'une construction géométrique basée sur les lois optiques ${ }^{27}$ ». Or, comme la découverte de ces lois et l'invention d'appareils de mesure connexes sont très complexes, il est normal que la représentation mimétique soit le fruit de développements graduels dont les étapes les moins abouties peuvent être qualifiées de primitives. Que Gombrich ne se contente pas de parler d'antériorité est symptomatique de sa position normative. Dans le cas des arts tridimensionnels, poursuit-il, la troisième dimension n'a pas besoin d'être conquise par une technique sophistiquée, puisqu'elle est donnée d'emblée et peut parfaitement être suggérée par des détails caractéristiques (une patte, une oreille, une corne).

Figure 4

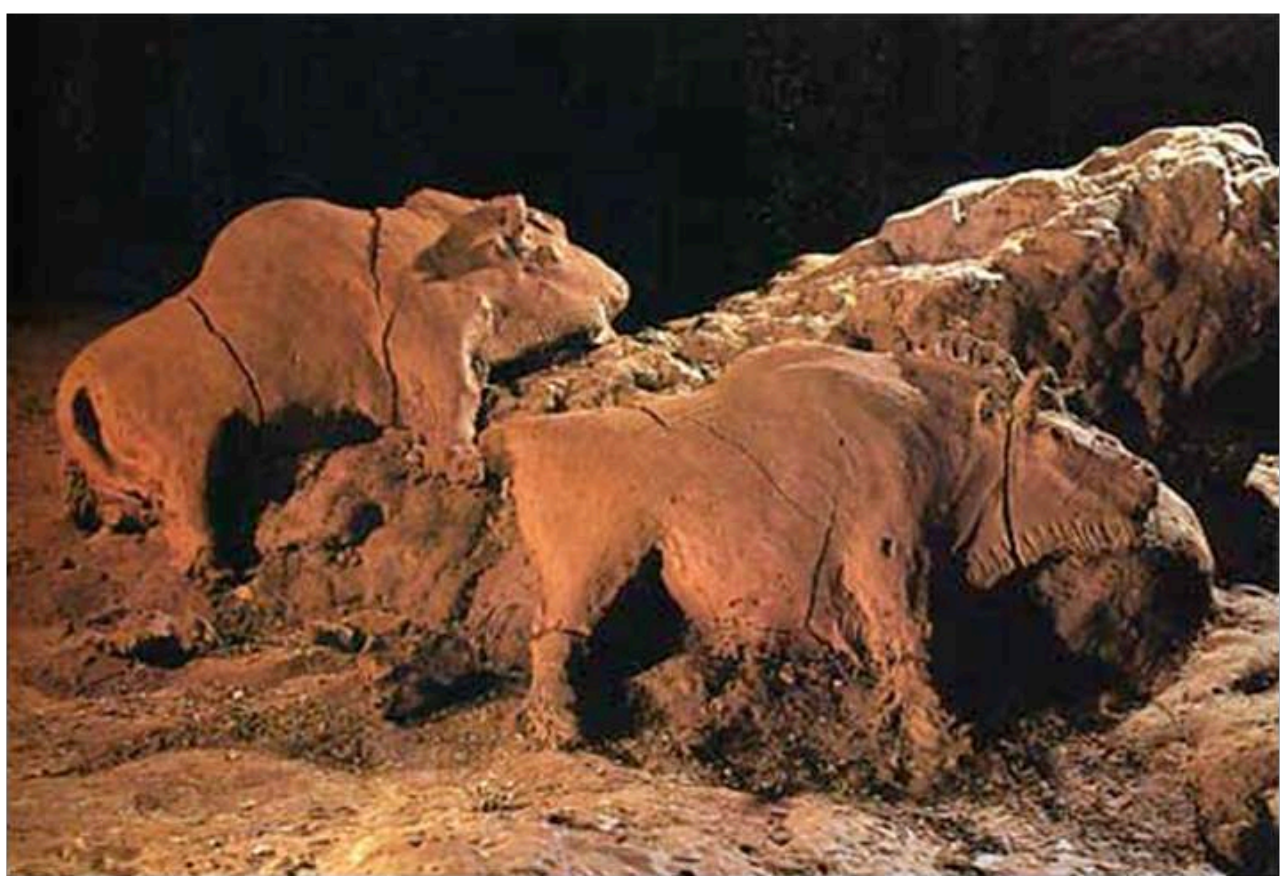

GROTTE DU TUC D'AUDOUBERT, MONTESQUIEU-AVANTÈS, ARIÈgE. BISONS. SCULPTURE SUR ARgILE. LONGUEUR DES DEUX BISONS : 61 ET 63 CM. MAgDALÉNIEN MOYEN.

12 Beaucoup d'arguments sont opposables à cette analyse, puisqu'on peut douter aussi bien de la valeur normative de l'imitation que de la définition que Gombrich en donne. Concernant le premier point, qu'il suffise d'évoquer la distinction que fait Panofsky, dans son analyse de la perspective, entre facteur du style et facteur de valeur esthétique ${ }^{28}$. Concernant le second, à savoir la conception gombrichienne de la mimésis comme restitution optiquement, c'est-à-dire scientifiquement, exacte de la perception - « Il y a une, mais seulement une solution correcte ${ }^{29}$ »-, rappelons simplement qu'elle est aussi concevable comme un processus d'idéalisation (Winckelmann). On pourrait enfin apporter des arguments de fait et remarquer que la stricte application des lois optiques en peinture est exceptionnelle, qu'il coexiste différents systèmes perspectifs ou encore que, dès la préhistoire, des techniques picturales de rendu perspectif ont été 
développées. L'art de la grotte Chauvet, dit Clottes, «atteint d'emblée un apogée par le naturalisme des représentations, la maîtrise des techniques, la richesse de l'invention. Ainsi, la perspective spatiale est rendue par divers moyens ${ }^{30} \%$.

Figure 5

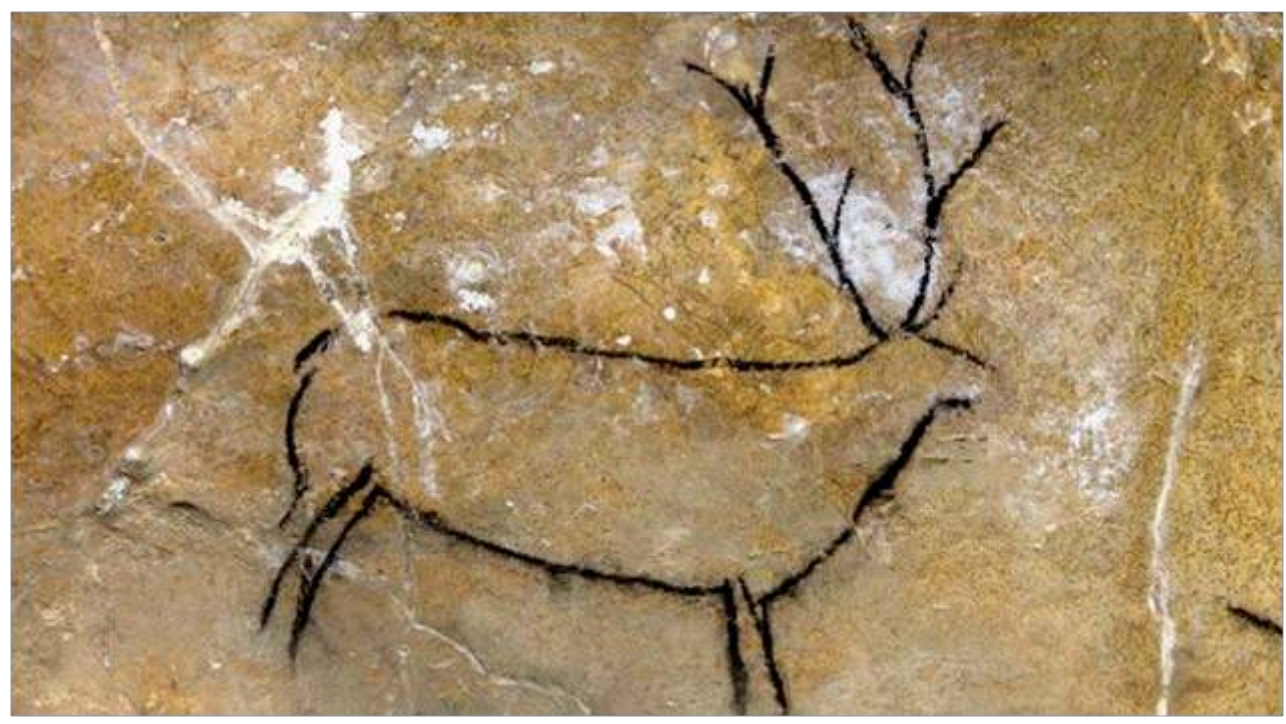

Grotte de Las Chimenas, Monte Castillo, Puente Viesgo, Cantabrie (Espagne). Cerf noir entier. Dessin au charbon sur roche. Longueur : environ $60 \mathrm{~cm}$. Solutréen ou Magdalénien.

Gombrich se rend bien compte du caractère arbitraire de sa reconstruction, mais n'en modifie pas pour autant ses conclusions. C'est un accident de l'histoire, dit-il, si nous avons tendance à considérer les styles antérieurs par le mauvais bout de la lorgnette, c'est-à-dire à vouloir expliquer pourquoi ils ont dévié de la mimésis alors que ce qui requiert des explications n'est pas l'absence mais l'invention de la mimésis ${ }^{31}$. Au lieu de s'attacher à cette question, Gombrich étudie les progrès de la représentation réaliste, excluant de l'histoire de l'art tout ce qui s'écarte de ce continuum téléologique pour l'assigner à la psychologie et à la nature. Les caractéristiques de l'art primitif, qu'il croit retrouver aussi bien dans les peintures de Lascaux que dans les dessins d'enfant, sont: la simplicité et la réduction des informations complexes, la représentation fragmentaire et schématique, la standardisation des distances et le peu d'indications de position, l'absence de troisième dimension, de modelé et de clair-obscur et la réduction des différentes parties du corps à des formules. Ces caractères sont négatifs et, c'est le point qui m'intéresse, anhistoriques.

Une fois qu'on a établi cette uniformité ou cette loi de transformation des images mimétiques, rien n'empêche de parler d'une parenté structurelle entre des images diverses que l'on peut qualifier de moins sophistiquées ou de primitives $^{32}$.

Poser une parenté structurelle entre ces différentes formes de primitivisme empêche toute appréhension stylistico-historique de leur spécificité. L'analyse par Gombrich de l'arc de Constantin (dont la valeur esthétique ne dépasse pas, selon lui, celle de moules à pain d'épices) montre particulièrement bien comment cette hypothèse d'une loi de transformation des images mimétiques fait obstacle à l'étude de la singularité historique de ce monument. Son absence de naturalisme (ses figures alignées sont réduites à des (stéréo)types, sa surface manque d'articulation) et le passage qu'il effectue de la sculpture antique classique, naturaliste, à un art statique posent 
évidemment problème à l'historien qui croit en un développement de l'habileté représentative. Gombrich refuse néanmoins de l'expliquer en termes de déclin et déclare simplement que ces artisans (il se garde de parler d'artistes!) «n'avaient pas besoin de voler et sont donc restés près du sol». (Il a développé plus haut une comparaison entre l'art aéronautique et l'art mimétique.) Plus que sur cette comparaison dépréciative, j'aimerais insister sur le fait que Gombrich écarte les explications historiques - «Les raisons sociales et historiques qui ont conduit à ce changement de fonction et de style [...] ne peuvent être notre objet »- au profit d'une argumentation psychologique et finalement naturaliste: c'est l'existence d'une loi psychologique de réduction des images naturalistes de trois à deux dimensions, comparable à l'action de la loi de gravitation, qui justifie qu'on qualifie «certaines caractéristiques structurelles des images » de primitives ${ }^{33}$. L'insistance sur l'uniformité structurelle des productions "primitives» (qui oublie que les ressemblances entre deux objets ne permettent pas nécessairement de conclure à leur identité) a pour conséquence la méconnaissance de leur ancrage culturel, esthétique, religieux, bref historique. Il semble donc y avoir pour Gombrich, d'une part, les productions mimétiques, que l'on peut ordonner historiquement et dater en fonction de leur perfection représentative et, d'autre part, les productions primitives (l'art de l'Antiquité tardive, l'art populaire, tribal, préhistorique, l'art des personnes sans instruction et des enfants) qui, analysables en termes psychologiques, sont exclues de l'histoire. Primitif signifie ici "non réaliste", c'est-à-dire non seulement ce qui ne correspond pas à la norme (n'est pas conforme), mais ce qui ne peut être jugé par rapport à elle, bref ce qui est hors-norme. Et dans la mesure où l'histoire de l'art est celle du développement de la représentation mimétique, elle en écarte le primitif. À la norme mimétique (historique) fait donc face le fait naturel (primitif). Si une pensée esthétique normative, qui voit dans la mimésis une valeur absolue, rend impossible toute considération artistique des œuvres préhistoriques, l'argumentation en termes psychologiques empêche leur insertion dans l'histoire.

\section{2.}

Des difficultés qu'il y a à penser l'art préhistorique dans l'histoire de l'art témoignent aussi les réflexions d'André Malraux. La définition de l'art à l'œuvre dans le musée imaginaire permet pourtant d'intégrer les créations préhistoriques au flux de l'art mondial. L'art, en effet, n'y est défini ni en termes d'adéquation à une essence (la beauté, l'harmonie, l'idéal) ni en termes fonctionnels. L'émergence de sa notion est bien plutôt pensée comme un phénomène historique, non pas simplement parce qu'elle apparaît à un certain moment (en l'occurrence avec la fin du christianisme), mais parce qu'elle correspond à l'avènement de la civilisation de l'histoire. Avec celle-ci, les cultures, et tout particulièrement les religions, perdent leur valeur d'absolu. Il est désormais possible d'unir ce qui était auparavant distingué, la sensibilité à «l'appel véhément adressé par un chef-d'œuvre à d'autres chefs-d'œuvre ${ }^{34}$ " permettant de mettre sur le même plan les chevaux de Lascaux et une tête de cheval de Braque ou de Degas. La substitution du devenir à l'être, de l'historique au théologique, fait qu'on peut désormais voir dans les dieux étrangers, non plus des démons, mais des formes, non plus les figures d'une divinité ou des idoles, mais des statues. De ce point de vue, il n'est pas plus insolite de considérer une Vierge médiévale comme une œuvre d'art que la dame à la capuche de Brassempouy (figure 6). Quant à l'argument selon lequel les 
hommes préhistoriques auraient été dépourvus d'intention esthétique, il ne suffit pas à écarter leurs productions du champ de l'art car, d'une part, leur situation ne diffère pas de celle des artistes d'avant l'époque de l'art et, d'autre part, il est de toute façon impossible de reconstituer les intentions des créateurs. "Peu importe à notre temps son ignorance des dieux des cavernes, et que l'idée d'art ait été étrangère aux Magdaléniens ${ }^{35}$ ». Le refus de faire de l'art une activité foncièrement mimétique (elle est un langage spécifique, non un moyen de représentation ou de suggestion) favorise, lui aussi, l'inscription des œuvres préhistoriques dans l'art universel. Ramener, comme le fait Gombrich, l'histoire de l'art à celle de la mimésis est insuffisant ${ }^{36}$.

Figure 6

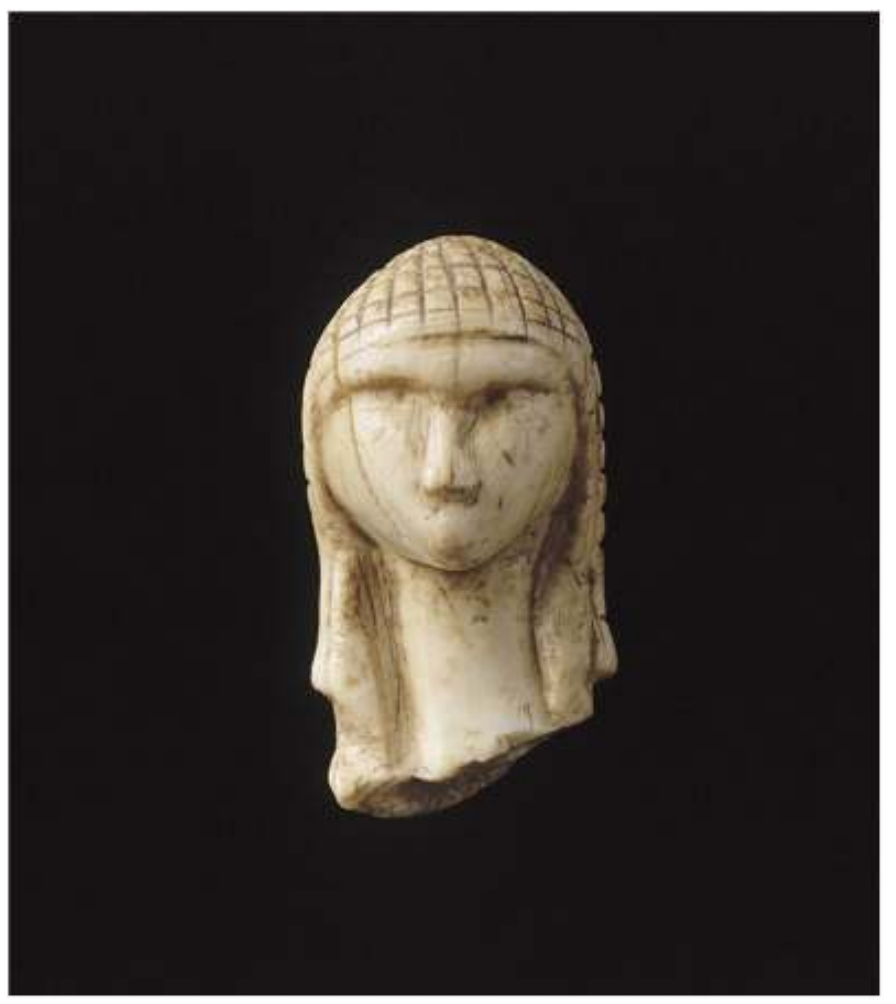

Dame à la Capuche (dite aussi la Dame de Brassempouy ou la Vénus de Brassempouy). Sculpture sur ivoire. Hauteur : $3,65 \mathrm{~cm}$. Découverte dans la grotte du Pape, Brassempouy, Landes ; conservée au musée d'Archéologie nationale, Saint-Germain-en-Laye, Gravettien.

Mais si Malraux reconnaît indéniablement aux peintures d'Altamira, de Lascaux, de Rhodésie le statut d'œuvre d'art, la Psychologie de l'art les écarte de l'histoire. L'argumentation de Malraux repose sur une distinction entre deux civilisations, l'une orientée vers l'éternité et l'autre, qui lui succède, vers l'histoire. "Mais l'éternité se retira du monde. [...] on finit par lui substituer le seul ennemi que l'esprit lui ait trouvé : l'histoire ». L'homme, désormais, n'est plus pensé comme « orienté vers l'Être » mais comme « orientable par des idées, par des actions » (c'est-à-dire comme libre) ${ }^{37}$. Il convient de signaler que si la production artistique sous le régime de l'éternité est étrangère à l'histoire, elle ne l'est pas à la temporalité ; l'art n'y est pas immobile et connaît des évolutions stylistiques. Mais de cette distinction entre sociétés de l'éternité et de l'histoire - perspective qui ne doit pas être confondue avec celle de Warburg qui pense bien plutôt la résurgence anachronique de l'éternel dans l'histoire -, faut-il conclure que les arts préhistorique, égyptien ou encore médiéval appartiennent à une 
seule et même catégorie et qu'une coupure radicale scinde l'art d'avant et d'après le XIX ${ }^{\mathrm{e}}$ siècle?

Une telle dichotomie est tout d'abord suggérée par le fait que, contrairement à Gombrich, Malraux n'isole pas l'art préhistorique. Il suppose par exemple que l'accent des monnaies celtiques remonte à la préhistoire et qu'il est peut-être celui des grands nomades $^{38}$. L'achèvement de la préhistoire ne signifie donc pas une rupture définitive de la tradition. Rupture, en revanche, il y a au XIX siècle (et non à la Renaissance), «le machinisme n'[ayant] peut-être pas d'autre précédent que la découverte du feu ${ }^{39}$ ». " Notre ressemblance avec Alexandrie ne pèse pas lourd en face d'un monde amputé en cent ans de rêves qu'il maintenait depuis le temps des cavernes ${ }^{40}$ ». L'industrialisation, la mécanisation marquent un changement de régime spirituel, le fait artistique nouveau étant que l'art n'a désormais plus d'autre fin que lui-même.

Deux éléments néanmoins remettent en question cette périodisation. D'une part, la rupture du $\mathrm{xIX}^{\mathrm{e}}$ siècle n'est pas absolue, la conception propre à «ce qui n'est pas seulement hors de l'histoire, mais hors du temps ${ }^{41}$ » ressurgissant à l'ère historique, dans les arts populaires notamment. On la retrouve aussi chez le douanier Rousseau dont Malraux pense que « ce qu'il peint de plus grand est lié à un passé sans histoire ${ }^{42}$ ». D'autre part, le continuum de la préhistoire au XIX siècle est mis à mal par l'introduction d'une distinction, au sein des civilisations de l'éternel, entre cultures primitives et civilisées. Si Malraux se refuse à qualifier les premières de sauvages («Peut-être la sauvagerie complète n'a-t-elle pas d'art du tout»), il considère néanmoins que «les civilisations dites primitives s'opposent à l'homme civilisé, mais aussi à l'homme historique ${ }^{43}$ ». Les primitifs selon Malraux (Cro-Magnon, l'Africain, l'Océanien) ont peu à voir avec nous, dont la civilisation commence avec l'Égypte des pharaons. Or, si Malraux, à l'encontre de sa distinction entre histoire et éternité, considère que ce sont « les pyramides ", figures de l'éternel s'il en est, qui symbolisent le commencement de notre culture (historique) ${ }^{44}$, c'est pour en séparer le primitif. Alors que sa périodisation initiale suggère une continuité de la préhistoire jusqu'au XIX siècle, il fait basculer l'art de l'Égypte jusqu'au xix siècle dans l'histoire pour en exclure d'autant mieux ce qu'il nomme le magdalénien. Lorsque il déclare que certains crucifix bretons de bois ne sont chrétiens qu'en apparence et expriment bien plutôt un sentiment multimillénaire de la vie qui s'est glissé dans une forme chrétienne (« c'est l'art d'une humanité qui prend les formes de l'histoire comme la lumière indifférente de la lune profile les palais successifs des hommes $\left.{ }^{45} »\right)$, il intègre l'art chrétien aux arts historiques alors même que la disparition de la chrétienté est censée coïncider avec leur avènement. L'analogie qui clôt son analyse : «il y a entre les arts de celle-ci [de l'histoire] et ceux qui lui sont étrangers, la même irréductible différence qu'entre tous les royaumes et l'époque des cavernes ${ }^{46}$ " montre qu'il distingue en réalité trois moments: l'historique, l'éternel et l'anti-historique (le primitif) et qu'il tend à rapprocher les deux premiers pour mieux les séparer du troisième.

Or cet autre que Malraux exclut selon des critères temporels mais aussi - dans le cas de l'Océanien et de l'Africain -, géographiques ${ }^{47}$, il refuse de le comprendre dans l'histoire, allant jusqu'à introduire la déroutante notion d'anti-histoire pour le qualifier.

[...] si le préhistorien obéit au même admirable effort d'élucidation du monde que l'historien, ce qu'il trouve n'est pas ce que cherchait celui-ci. La préhistoire n'est pas une histoire diffuse : c'est une anti-histoire, et l'homme que nous y pressentons, comme souvent l'Africain et l'Océanien, est anti-historique ${ }^{48}$. 
Concevoir l'étude de la préhistoire comme une remontée vers les origines, peut-être même biologiques, de l'homme est erroné, puisqu'une différence fondamentale nous coupe d'eux. Parler d'anti-histoire et non de préhistoire souligne que celle-ci est moins ce qui vient avant notre civilisation que ce dont nous nous séparons. Certes, le primitif ne saurait être considéré comme un sous-homme, mais il appartient à une autre humanité. "L'homme étranger à l'histoire, ne nous apparaît plus nécessairement derrière l'homme historique - voire en face de lui - comme un déchet, mais comme un autre type d'humain ${ }^{49}$ ». Le préhistorique, ensuite, se voit écarté de la civilisation.

Les temps sans histoire (la proto-histoire, le "grand temps ») ne connaissent pas seulement les crânes de buffles au bout des lances, les chiffons accrochés aux arbres de montagne, mais aussi leurs propres styles, ceux des cavernes par exemple ; alors se dissipe l'illusion qui fait de toutes les œuvres sauvages les œuvres de l'instinct. Et nous commençons à découvrir que le style n'est pas seulement lié à ce que nous appelons la civilisation, ni même aux « cultures $»^{50}$.

En écartant l'idée que les peintures d'Altamira ou de Lascaux sont le fruit de l'instinct, Malraux reconnaît qu'elles relèvent de l'art («Il n'y a pas de style de l'expression instinctive $\left.{ }^{51} »\right)$. Mais il leur refuse l'appartenance à la civilisation. Or, si cet art n'est le fruit ni de la nature (instinct) ni de la culture, on ne voit pas bien d'où il peut venir. Euvres de l'art («les bisons d'Altamira [...] sont réellement des œuvres élaborées » (figure 7); «les peintures magdaléniennes n'appartiennent pas à l'informe»), les œuvres préhistoriques sont pourtant hors de l'histoire et appartiennent à «la zone de limbes qui avait été celle du monde préhellénique ${ }^{52} »$. Bref l'art préhistorique se voit exclu tant de l'histoire que de la civilisation, et ce qui vaut pour lui vaut du primitif en général ${ }^{53}$.

\section{Figure 7}

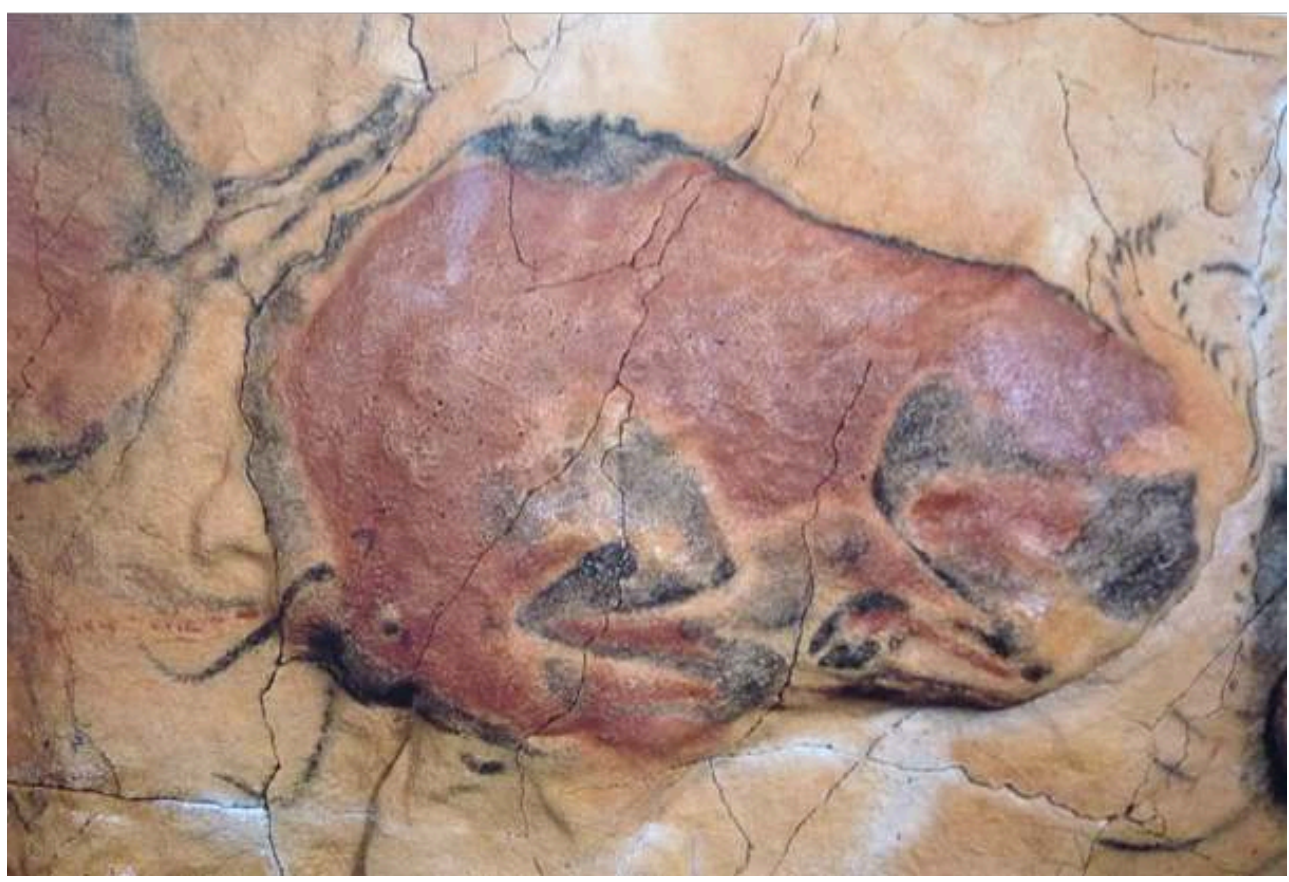

Grotte d'Altamira, Santillana del Mar, Cantabrie. Bison recroquevillé sur une bosse. Grand plafond. Peinture sur roche. Magdalénien inférieur / moyen. 
D'autres réflexions de Malraux néanmoins semblent difficilement conciliables avec ces conclusions. L'ensemble des œuvres que nous qualifions d'artistiques présente une parenté psychologique, pour ne pas dire métaphysique, dans la mesure où elles sont liées à un commun combat contre le destin. «Toute œuvre qui nous donne le sentiment de la qualité artistique relie aussi au monde les profondeurs qu'elle exprime; toute œuvre qui nous atteint par là témoigne d'une part victorieuse de l'homme, fût-il un homme fasciné ${ }^{54}$ ». Or cet homme fasciné ne désigne-t-il pas aussi le magdalénien ? Ce ne sont donc pas aux seules couleurs et formes du passé que nous sommes sensibles, mais aussi à la lutte qui s'y joue et dans laquelle l'homme prend conscience de luimême et de ses pouvoirs. Tout comme l'artiste de génie crée des formes qui lui permettent de posséder un univers autonome, cohérent et significatif en arrachant de nouvelles formes à l'héritage historique (la clef de la création étant donc moins la tradition que sa rupture), l'art est une victoire sur le destin de l'humanité. « L'art est un anti-destin » :

une mosaïque byzantine, un Rubens, un Rembrandt, un Cézanne : toutes ces œuvres (...) s'unissent aux peintures magdaléniennes dans le langage immémorial de la conquête, non dans un syncrétisme de ce qui fut conquis ${ }^{55}$.

Or comment tenir ensemble l'idée que les primitifs appartiennent à une autre humanité et celle qu'une même communauté humaine nous relie à eux? Les remaniements auxquels procède Malraux entre les deux éditions de la Monnaie de l'absolu prennent acte de cette difficulté et, au prix d'une réélaboration de la conception du temps et de l'histoire, accueillent complètement l'art magdalénien.

De fortes constantes relient évidemment les deux textes, mais leurs variations sont essentielles pour le problème qui nous occupe. Il faut tout d'abord souligner que Malraux n'écarte plus le préhistorique de l'histoire. Alors que La psychologie de l'art en faisait une "anti-histoire ", les Voix du silence y voient " une autre histoire » qui, en outre, n'est pas coupée des autres. La culture océanienne par exemple pourrait bien être une survivance de l'âge des mégalithes ${ }^{56}$. Remarquable aussi est que Malraux ne parle désormais plus de ce qui est hors du temps, mais de ce qui «semble hors du temps ${ }^{57}$ ». Intégré à l'histoire, l'homme "pré »historique l'est aussi complètement à l'humanité. Non seulement Malraux renonce à voir une rupture de continuité entre l'homme de Lascaux et celui des pyramides, mais il va jusqu'à inclure dans l'humanité les cousins de l'homme en évoquant « la première nuit glacée où une sorte de gorille se sentit mystérieusement le frère du ciel étoilé. Il est l'éternelle revanche de l'homme ${ }^{58}$ ». Dans Les voix du silence, Malraux tire donc toutes les conséquences de la métamorphose. La création artistique est un concept moderne qu'il est vain de prétendre éclairer par une reconstitution des intentions et des sentiments des créateurs. «La foi que nous transmettent les œuvres médiévales n'est plus tout à fait la leur. Elle nous parvient à travers la métamorphose, dont l'action devient plus visible lorsqu'elle touche un plus lointain passé ». L'impossibilité de penser et de sentir comme l'homme d'Altamira est un cas extrême, non un cas particulier de l'impossible reconstitution du sens originel des œuvres que nous considérons comme artistiques. "L'Occidental de la seconde moitié $\mathrm{du} \mathrm{xIx}^{\mathrm{e}}$ siècle était aussi étranger à l'ancienne Égypte qu'aux temps des cavernes ». La métamorphose a également pour conséquence que l'art ne nous livre que peu d'informations sur une civilisation. «Le bison d'Altamira [...] ne nous enseigne rien (à l'exception de la présence de la création artistique jusque dans la préhistoire) des Magdaléniens, et peu de chose du sentiment magique particulier qu'il exprime ${ }^{59}$ ». Et 
cela est d'autant plus vrai que l'art est un reflet déformé, ou mieux embelli, de son temps ( « Si atroce que soit un temps, son style n'en transmet jamais que la musique; le musée imaginaire est le chant de l'histoire, il n'en est pas l'illustration ») et qu'une reconstruction historique exhaustive est illusoire ( «l'étendue de notre musée imaginaire suffit à rendre superficielles les connaissances historiques qu'il appelle $\left.{ }^{60} »\right)$.

Que notre conception de l'art comme anti-destin soit issue de sa métamorphose n'est pas sans conséquence sur la manière d'écrire l'histoire. D'abord, parce que ce concept d'art nous est propre, on ne peut considérer qu'il constitue la fin (le but) des artistes dans l'histoire. Ensuite, parce que l'histoire de l'art est celle d'actes de libération, elle ne peut être réduite à un enchaînement de relations historiques objectives (« Lorsque les civilisations du passé s'ordonnent à nos yeux selon leurs valeurs propres, la Raison pèse sur Robespierre comme le Christ sur saint Louis $\left.{ }^{61} »\right)$. Pour retracer l'histoire de l'art, il ne faut donc ni la concevoir téléologiquement à partir de son terme prétendu ni l'envisager de manière causale en référence à une origine à partir de laquelle se déploierait une série d'effets, mais la remonter, comprendre contre quoi (contre quelle tradition, quel présent) l'artiste crée ses formes. Les artistes qui « inventent un système de formes, ne l'arrachent ni à leur soumission à la nature, ni à leur seul sentiment, mais le doivent à leur conflit avec une autre forme d'art ${ }^{62} »$. Seule cette histoire, qui ne se confond ni avec une construction téléologique ni avec une succession d'influences, est compatible avec l'affirmation de la liberté de l'homme et la définition de l'art comme lutte contre le déjà-donné. Il en résulte aussi que la considération historique de l'art passe in fine à côté de l'essentiel. "Ce que nous savons du Japonais du XII siècle est irréductible à l'évidence qui nous fait reconnaître dans les portraits de Takanobu l'un des sommets de la peinture mondiale». Tandis que le premier genre de connaissance est historique, le second est psychologique. Si nous pouvons nous reconnaître dans l'art japonais du XII ${ }^{\mathrm{e}}$ siècle, ce n'est pas parce que nous avons accumulé des informations historiques à son propos, mais parce que nous reconnaissons son appartenance à « une invincible part du présent artistique ${ }^{63} »$.

L'intégration du préhistorique dans l'histoire implique donc l'abandon de la tripartition entre primitif, éternel et historique au profit de l'idée d'une coulée humaine.

L'immense domaine d'art qui monte pour nous du passé n'est ni éternel, ni audessus de l'histoire ; il est lié à celle-ci et lui échappe à la fois comme Michel-Ange à Buonarroti. Son passé n'est pas un temps révolu, mais un possible ; il n'impose pas une fatalité, il établit un lien ${ }^{64}$.

Si le magdalénien n'incarne plus une altérité irréductible, ce n'est pas parce qu'il serait possible de le situer exactement dans l'histoire et de tracer la généalogie qui le relie jusqu'à nous - Malraux ne voit d'ailleurs aucunement dans l'émergence de la conscience historique une sorte d'esprit absolu, la «raison» et l'«histoire» appartenant bien plutôt à ces valeurs éphémères qui accompagnent une civilisation -, mais en vertu d'une permanente métamorphose.

La succession des cultures ne se syncrétise pas en quelque théosophie culturelle, parce que l'humanité se continue selon la plus profonde des métamorphoses, et non grâce à des adjonctions, ni même à une croissance : Athènes n'est pas l'enfance de Rome - Sumer moins encore. [...] Nous pouvons tout unir, sauf l'essentiel ${ }^{65}$.

Nous ne sommes donc pas proches de Lascaux parce que nous en serions les successeurs, mais parce que nous appartenons à la même vague d'humanité. " Nous ne cherchons pas dans certaines de leurs valeurs la "préfiguration " des nôtres: nous sommes moins héritiers de telle ou de telle en particulier, ou de toutes juxtaposées, que 
de leur ensemble et singulièrement de la coulée profonde qui les suscita ${ }^{66} »$. Ce qui fait la force d'une œuvre d'art n'est pas que son influence subsiste jusqu'à nous, mais que nous puissions y reconnaître ce noyau d'humanité. «La survie ne se mesure pas à la durée ; elle est celle de la forme que prit la victoire d'un homme sur le destin, et cette forme, l'homme mort, commence sa vie imprévisible ${ }^{67}$ ». Cette coulée humaine, enfin, n'est ni homogène ni donnée: notre culture n'est pas faite de passés inventoriés et conciliés, mais de parts inconciliables du passé. «Le passé se conquiert; [...] c'est en nous, par nous, que devient vivant le dialogue des ombres ${ }^{68} »$. La prise en compte du fait préhistorique implique donc un réexamen de nos catégories temporelles usuelles et rend caducs les schémas historiques linéaires et continus, strictement chronologiques, téléologiques et tendanciellement idéologiques véhiculés par une certaine histoire de l'art.

\section{BIBLIOGRAPHIE}

Belleforest François (de), « La cosmographie universelle de tout le monde », 1575.

Boas Franz, Primitive Art, Cambridge Mass., Harvard University Press, 1927 (il existe une traduction française par Marie Mausé, voir Boas, Franz, L'art primitif, Paris, Éditions Adam Biro, 2003.)

Clottes Jean, L'art des cavernes, Paris, Phaidon, 2008.

Clottes Jean, Pourquoi l'art préhistorique?, Paris, Gallimard, 2011.

Gombrich Ernst Hans, Geschichte der Kunst [1950], Cologne, Kiepenheuer \& Witsch, 1959. Pour la traduction française, voir Ernst Hans Gombrich, Histoire de l'art, Jacques Combe, Claude Lauriol et Dennis Collins (trad.), Phaidon, 2001, 16 édition.

Gombrich Ernst Hans, L'art et l'illusion, Psychologie de la représentation picturale [1960], Guy Durand (trad.), Paris, Gallimard, 1996.

Gombrich Ernst Hans, The Preference for the Primitive. Episodes in the History of Western Taste and Art (posthume), Londres, Phaidon, 2002. Pour la traduction française, voir Ernst Hans Gombrich, La préférence pour le primitif. Épisodes d'une histoire du goût et de l'art en Occident, Dominique Lablanche (trad.), Londres, Phaidon, 2004.

Klibansky Raymond, Panofsky Erwin, Saxl Fritz, Saturne et la mélancolie. Études historiques et philosophiques : nature, religion, médecine et art [1964], Fabienne Durand-Bogaert et Louis Evrard (trad.), Paris, Gallimard, 1989.

Malraux André, Psychologie de l'art : Le Musée imaginaire, La Création artistique, La Monnaie de l'absolu [noté PA], Paris, Albert Skira Éditeur, 1947-1950, 3 vol. Remaniés pour devenir, en 1951, Les Voix du silence [noté VS]. Elles comprennent quatre parties : Le musée imaginaire, Les métamorphoses d'Apollon, La création artistique et La monnaie de l'absolu.

Panofsky Erwin, « La perspective comme forme symbolique » [1927], in La perspective comme forme symbolique et autres essais, traduction sous la direction de Guy Ballangé, Paris, Minuit, 1975. 
Panofsky Erwin, La sculpture funéraire de l'ancienne Égypte au Bernin [1964], Dennis Collins (trad.), Paris, Flammarion, 1995.

Riegl Alois, Questions de style. Fondements d'une histoire de l'ornementation [1893], Henri-Alexis Baatsch et Françoise Rolland (trad ;), Paris, Hazan, 1992.

Rivière Émile, « Note sur l'ordre chronologique véritable des six premières découvertes de grottes à gravures et à peintures ", in Bulletin de la Société préhistorique française, 1909, t. 6, $\mathrm{n}^{\circ} 7$, p. 376-380. Disponible en ligne : http://www.persee.fr/web/revues/home/prescript/article/ bspf_0249-7638_1909_num_6_7_7945.

Roussot Alain, « Les premières découvertes d'art pariétal », in Paléo, hors-série, 1990. Disponible en ligne : http://www.persee.fr/web/revues/home/prescript/article/

pal_1145-3370_1990_hos_1_1_1415.

Vasari Giorgio, Les vies des meilleurs peintres, sculpteurs et architectes, traduction et édition sous la direction d'André Chastel, Arles, Actes Sud, 2005 (2 vol.), t. 1.

Warburg Aby, « Souvenirs d'un voyage en pays pueblo ». Notes inédites pour la conférence de Kreuzlingen sur « Le rituel du serpent » [1923], in Philippe-Alain Michaud, Aby Warburg et l'image en mouvement, Paris, Macula, 1998.

\section{NOTES}

1. Je reprendrai ici la définition et la périodisation de l'art préhistorique proposées par Jean Clottes dans L'art des cavernes, Paris, Phaidon, 2008, p. 11. Il s'y concentre sur l'art paléolithique européen qui n'est pas le plus ancien, mais le mieux connu et le mieux daté. Il couvre une période de 20000 à 25000 ans (de l'aurignacien au magdalénien) et s'achève avec la fin de la dernière glaciation, il y a près de 11000 ans. Concernant les productions des populations antérieures (Homo erectus et Néanderthaliens), Jean Clottes hésite à les qualifier d'œuvres d'art si l'on entend par là ce qui « résulte de la projection, sur le monde qui entoure l'homme, d'une image mentale forte qui colore la réalité, avant de prendre forme et de la transfigurer ou de la recréer dans la matière » (ibid.).

2. Giorgio Vasari, Les vies des meilleurs peintres, sculpteurs et architectes, traduction et édition sous la direction d'A. Chastel, Arles, Actes Sud, 2005 (2 vol.), tome 1, préface aux Vies, p. 215 : «C'est en s'inspirant de l'homme [...] comme d'un vrai modèle, que peu à peu furent composées par la suite statues et sculptures ».

3. Émile Rivière, «Note sur l'ordre chronologique véritable des six premières découvertes de grottes à gravures et à peintures ", in Bulletin de la Société préhistorique française, 1909, tome 6, n 7 , p. 376-380. Disponible en ligne: http://www.persee.fr/web/revues/home/prescript/article/ bspf_0249-7638_1909_num_6_7_7945

4. Dès la fin du $\mathrm{XvI}^{\mathrm{e}}$ siècle par exemple, François de Belleforest évoque, dans «La cosmographie universelle de tout le monde » (1575), les ornements des grottes du Périgord (vraisemblablement de Rouffignac, «redécouvertes" en 1956). Il mentionne quelques autels et des peintures en plusieurs endroits, mais sans, bien sûr, pouvoir les rapporter à la préhistoire. De même, au XVII ${ }^{\mathrm{e}}$ siècle, un érudit sarladais, le chanoine Tarde, déclare avoir vu dans cette grotte « des peintures en plusieurs lieux, montrant des vestiges de toutes sortes de bétail », mais il n'établit aucun lien avec une culture préhistorique, notamment pour des raisons théologiques. Pour ces deux exemples et l'impossibilité, pour les découvreurs, de penser «l'existence d'un homme préhistorique, et partant [...] ses productions artistiques », voir Alain Roussot, «Les premières 
découvertes d'art pariétal ", in Paléo, hors-série, 1990, p. 34-35. En ligne : http://www.persee.fr/ web/revues/home/prescript/article/pal_1145-3370_1990_hos_1_1_1415.

5. Alois Riegl, Questions de style. Fondements d'une histoire de l'ornementation [1893], traduction H.A. Baatsch et F. Rolland, Paris, Hazan, 1992, chapitre 1, p. 28-29.

6. Jean Clottes, L'art des cavernes, p. 24. Pour lui, l'hypothèse chamanique rendrait actuellement le mieux compte des faits connus. Voir aussi Pourquoi l'art préhistorique?, Paris, Gallimard, 2011, chap. 1, p. 34 , où il précise que cette hypothèse, émise par Mircea Eliade au début des années 1950, a été considérablement développée, les 25 dernières années, par David Lewis-William. Elle ne se substitue pas aux autres théories avancées (théorie de l'art pour l'art, totémisme, magie de la chasse, structuralisme) mais intègre nombre de leurs éléments (Ibid., conclusion, p. 276-281).

7. Ernst Hans Gombrich, Geschichte der Kunst [1950], Cologne, Kiepenheuer \& Witsch, 1959, chap. 1: «Étranges débuts: l'art de la préhistoire, des primitifs et de l'Amérique avant sa découverte ». C'est moi qui traduis. Pour la traduction française, voir Ernst H. Gombrich, Histoire de l'art, traduction de Jacques Combe, Claude Lauriol et Dennis Collins, Phaidon, 2001, $16^{\mathrm{e}}$ édition.

8. Jean Clottes, Pourquoi l'art préhistorique?, chap. 1, p. 37. Conscient des limites d'une analogie dont Claude Lévi-Strauss et André Leroi-Gourhan ont souligné les dangers, Clottes estime néanmoins qu'il est possible et nécessaire d'entreprendre un comparatisme ethnologique. Celuici ne consiste ni à calquer une réalité moderne sur le passé ni à établir des analogies ponctuelles, mais à « indique[r] des possibilités de concepts et de structures sociales et mentales voisines, ou encore des récurrences fréquentes de comportements et d'attitudes dans certains contextes » (p. 71), tel par exemple la croyance au pouvoir des images, fondement de la magie sympathique. Loin de postuler une mentalité primitive ou une humanité sauvage, cette approche repose sur la conviction que « l'humanité est une [...] et [que] les races n'existent pas ». "C'est cette réalité et ce sont ces évidences qui permettront d'extrapoler certains comportements humains connus à un passé qui l'est beaucoup moins» (p. 53-54). Voir aussi la section «Apports et risques de la comparaison ethnologique » (p. 68-74).

9. Gombrich fait sienne la théorie que Clottes nomme magie sympathique ou magie de la chasse. Voir L'art des cavernes, p. 23 : La magie sympathique se fonde sur une relation vitale entre l'image et son sujet. En agissant sur l'image, on agit sur la personne ou l'animal figuré. Cette théorie, répandue par l'abbé Henri Breuil et le comte Henri Bégouën, connut une fortune étonnante pendant toute la première moitié $\mathrm{du} \mathrm{xx}^{\mathrm{e}}$ siècle, sous le nom générique de " magie de la chasse ». L'interprétation selon laquelle ces dessins avaient pour but de rendre la chasse fructueuse, de détruire les animaux nuisibles et de multiplier le gibier est rejetée dans la seconde moitié du xx siècle.

10. Voir notamment Enrich H. Gombrich, L'art et l'illusion, Psychologie de la représentation picturale [1960], G. Durand (trad.), Paris, Gallimard, 1996, introduction, p. 16.

11. Enrich H. Gombrich, Geschichte der Kunst, chap. 1, p. 28.

12. Ibid., chap. 1, p. 29.

13. Voir Enrich H. Gombrich, The Preference for the Primitive. Episodes in the History of Western Taste and Art (posthume), Londres, Phaidon, 2002, chap. 7 : “Primitive - in what Sense?" (p. 269-297). Il existe une traduction française de l'ouvrage. Voir E. H. Gombrich, La préférence pour le primitif. Épisodes d'une histoire du goût et de l'art en Occident, Dominique Lablanche (trad.), Londres, Phaidon, 2004.

14. Enrich H. Gombrich, Die Geschichte der Kunst, chap. 1, p. 40.

15. Ibid., p. 42.

16. Ibid., chap. 2, p. 43.

17. Loc. cit.

18. Jean Clottes, L'art des cavernes, p. 28.

19. Ibid., p. 17 ainsi que pour l'évocation de ces différentes techniques. 
20. Sur le phénomène de pseudomorphose, voir Erwin Panofsky, La sculpture funéraire de l'ancienne Égypte au Bernin [1964], D. Collins (trad.), Paris, Flammarion, 1995, chap. 2, p. 31; sur celui de recréation spontanée, voir Raymond Klibansky, Erwin Panofsky et Fritz Saxl, Saturne et la mélancolie. Études historiques et philosophiques: nature, religion, médecine et art [1964], F. DurandBogaert et L. Evrard (trad.), Paris, Gallimard, 1989, IV partie, chapitre 2, p. 485.

21. Aby Warburg, «Souvenirs d'un voyage en pays pueblo». Notes inédites pour la conférence de Kreuzlingen sur « Le rituel du serpent » (1923), in Philippe-Alain Michaud, Aby Warburg et l'image en mouvement, Paris, Macula, 1998, p. 255.

22. Enrich H. Gombrich, The Preference for the Primitive, op. cit., chap.7, p. 297. C'est moi qui traduis.

23. Ibid., chap. 7, p. 297.

24. Ibid., chap. 5, p. 200.

25. Franz Boas, Primitive Art, Cambridge, Mass., Harvard University Press, 1927, voir préface, p. 1-4 pour l'exposé du premier principe, p. 2-7 pour l'exposé du second. Il existe une traduction française de l'ouvrage. Voir Franz Boas, L'art primitif, Marie Mauzé (trad.), Paris, Éditions Adam Biro, 2003. Sur l'identité de la structure mentale de l'homme moderne et du préhistorique, voir aussi Jean Clottes, L'art des cavernes, p. 11: «Il y a quarante mille ans environ, des nouveaux venus arrivèrent en Europe occidentale, région occupée depuis plus de deux cent mille ans par les Néanderthaliens. Ils venaient d'Europe centrale et orientale et provenaient du Proche-Orient, qu'ils avaient atteint, quelques dizaines de millénaires plus tôt, à partir de l'Afrique dont l'espèce humaine est originaire. On les a appelés les Cro-Magnons. C'étaient des gens comme nous, avec le même cerveau, le même aspect physique, le même système nerveux et les mêmes capacités que les nôtres ».

26. Enrich H. Gombrich, The Preference for the Primitive, chap. 7, p. 271.

27. Ibid., chap. 7, p. 277.

28. Erwin Panofsky, «La perspective comme forme symbolique » [1927], in La perspective comme forme symbolique et autres essais, traduction sous la direction de G. Ballangé, Paris, Minuit, 1975, II, p. 78. « Si la perspective n'est pas un facteur de la valeur artistique, du moins est-elle un facteur du style ».

29. Enrich H. Gombrich, The Preference for the Primitive, chap. 7, p. 279.

30. Jean Clottes, L'art des cavernes, p. 12. C'est moi qui souligne. Voici quelques exemples de perspective mentionnés par Clottes. À Chauvet, sur le panneau des rhinocéros, deux procédés créent une perspective spatiale. Sur le haut des pattes droites du rhinocéros central, situées au deuxième plan, un blanc a été réservé qui fait défaut aux pattes gauches plus proches de l'observateur, ce qui introduit un effet de décalage. Quant aux rhinocéros du haut, après qu'un animal entier ait été dessiné, deux cornes ont été ajoutées sur l'avant et trois sur l'arrière pour donner l'illusion d'un troupeau d'animaux placés côte à côte. La taille décroissante des cornes rend l'effet de perspective. Enfin, l'illusion est accentuée par les lignes de dos tracées parallèlement à celle de l'animal central (Ibid., p. 40). À Lascaux, la perspective spatiale des pattes est suggérée par le fait qu'elles ne sont pas reliées directement au corps, un blanc étant laissé au niveau de l'attache et parfois sur la surface entière de la patte au second plan (Ibid., p. 113). Dans la grotte de Las Chimenas, la patte postérieure gauche du grand cerf est attachée à la ligne interne de celle de droite, produisant un effet de perspective spatiale (Ibid., p. 165) (figure 5). Enfin, dans Pourquoi l'art préhistorique ?, chap. 3, p. 260, Clottes évoque l'une des vaches de Lascaux positionnée en haut de paroi, qui a été délibérément dessinée d'une manière non réaliste afin d'être vue avec des proportions parfaites par le spectateur situé plus bas.

31. Enrich H. Gombrich, The Preference for the Primitive, chap. 7, p. 279-280.

32. Ibid., chap. 7, p. 285.

33. Ibid., chap. 7, p. 291. 
34. André Malraux, Psychologie de l'art [noté PA], $3^{\mathrm{e}}$ vol. La monnaie de l'absolu, Paris, Albert Skira Éditeur, 1950, chapitre 3, p.90. On retrouve l'expression dans Les voix du silence [noté VS], $4{ }^{\mathrm{e}}$ partie : La monnaie de l'absolu, Paris, NRF Gallimard, 1951, IV, p. 556. Je cite à dessein les deux éditions dont les variations joueront un rôle dans mon argumentation. D'abord publiés chez Skira, les trois volumes de la Psychologie de l'art (1947-1950): Le Musée imaginaire, La Création artistique, La Monnaie de l'absolu, sont repris et remaniés pour devenir, en 1951, Les Voix du silence [noté VS]. Elles comprennent quatre parties : Le musée imaginaire, Les métamorphoses d'Apollon, La création artistique et La monnaie de l'absolu.

35. La monnaie de l'absolu (VS), II, p. 525

36. Voir par exemple Ibid., VI, p. 613 : «L'élaboration du style égyptien [...] n'est pas dans la conquête de la fidélité de ses portraits, qu'il partage avec beaucoup d'autres, mais dans la conquête de la frontalité ».

37. La monnaie de l'absolu (PA), chap. 2, p. 47 ; La monnaie de l'absolu (VS), I, p. 479-480.

38. La monnaie de l'absolu (PA), chap. 3, p. 53 ; La monnaie de l'absolu (VS), II, p. 500.

39. La monnaie de l'absolu (PA), chap. 5, p. 151 ; La monnaie de l'absolu (VS), VII, p. 638.

40. La monnaie de l'absolu (PA), chap. 3, p. 108 ; La monnaie de l'absolu (VS), V, p. 589. Notons que dans L'art des cavernes, p. 22, Jean Clottes voit lui aussi dans le machinisme du XIX siècle une rupture civilisationnelle qui justifie que, d'un point de vue ethnologique, on rapproche la culture préhistorique de celle des sociétés traditionnelles. Leur mode de pensée est en effet plus proche de celui des chasseurs-collecteurs d'autres continents que du nôtre, Occidentaux matérialistes vivant dans une société complexe de type industriel au début $\mathrm{du} \mathrm{xxI}^{\mathrm{e}}$ siècle. Une disposition d'esprit propre aux cultures traditionnelles, que l'on trouve aussi dans les manifestations paléolithiques, est par exemple la perméabilité ou fluidité (entre le monde habité et le monde surnaturel ou entre les hommes et les animaux). Sur l'usage du comparatisme ethnologique par le préhistorien, voir note 8.

41. La monnaie de l'absolu (PA), chap. 3, p. 93

42. La monnaie de l'absolu (PA), chap. 3, p. 67 ; La monnaie de l'absolu (VS), II, p. 507, qui ajoute une comparaison entre le cheval de La guerre du douanier Rousseau (vers 1894) et les chevaux magdaléniens : " le cheval de La Guerre est celui des peintures magdaléniennes ».

43. La monnaie de l'absolu (PA), chap. 3, p.91; La monnaie de l'absolu (VS), IV, p. 556 pour la première expression; La monnaie de l'absolu (PA), chap. 3, p. 91 pour la seconde.

44. La monnaie de l'absolu (PA), chap. 3, p. 92. Voir aussi Ibid., chap. 4, p. 125 : « Aussi brutalement que l'ère des machines rompt avec tout ce qui la précède, l'artiste du XIX ${ }^{\mathrm{e}}$ siècle se sépare de ceux qui étaient ses prédécesseurs depuis quatre mille ans ».

45. La monnaie de l'absolu (PA), chap. 3, p. 91 ; La monnaie de l'absolu (VS), IV, p. 558.

46. La monnaie de l'absolu (PA), chap. 3, p. 91-92.

47. Faire débuter notre civilisation en Égypte, en Méditerranée, c'est en quelque sorte la faire commencer en Europe. Et si Malraux insiste tant pour faire de l'industrialisation une césure civilisationnelle, c'est peut-être parce que, en 1950, celle-ci constitue encore un phénomène essentiellement européen (et nord-américain).

48. La monnaie de l'absolu (PA), chap. 3, p. 91.

49. Ibid., chap. 3, p. 95. La formule est reprise, modifiée, dans La monnaie de l'absolu (VS), IV, p. 560 où Malraux parle d'« un autre type humain ».

50. La monnaie de l'absolu (PA), chap. 3, p. 93-94.

51. Ibid., chap. 3, p. 95.

52. Voir Ibid., chap. 3, p. 94-95 pour ces trois citations. Dans Les voix du silence, la réflexion sur le caractère élaboré des œuvres préhistoriques apparaît dans la $3^{\mathrm{e}}$ partie : La création artistique, II, p. 303.

53. Ce qui vaut du magdalénien (c'est le terme que Malraux emploie généralement) vaut en effet de l'africain. Son art peut résulter d'une intention artistique et d'une volonté formelle - il 
advient que l'artiste africain, doué d'un véritable « sentiment esthétique » tente, dans l'évocation des figures d'ancêtres et d'animaux, "seulement d'en maîtriser les formes ». " Il advient que les noirs soient artistes parce qu'ils sont possédés, il advient aussi qu'ils le soient parce qu'ils sont des artistes ». Mais ces productions n'appartiennent pas à l'histoire - « ces sculptures de bois [...] ne font que frôler l'histoire» (La monnaie de l'absolu (PA), chapitre 3, p. 98). La formule est notablement modifiée dans Ibid. (VS).

54. La monnaie de l'absolu (PA), chap. 3, p. 197; la formule est reprise, avec une légère modification, dans La monnaie de l'absolu (VS), IV, p. 574.

55. La monnaie de l'absolu (PA), chap. 5, p. 151. L'expression est reprise avec des variations peu significatives dans Ibid. (VS), VII, p. 637.

56. La monnaie de l'absolu (VS), IV, p. 557.

57. Ibid., IV, p. 559. Je n'ai pas trouvé dans l'édition de 1951 la remarque, déjà évoquée, selon laquelle « les civilisations dites primitives s'opposent à l'homme civilisé, mais aussi à l'homme historique ». Elle devrait en toute logique se trouver dans Ibid. (VS), IV, p. 556.

58. La monnaie de l'absolu (VS), VII, p. 635.

59. Ibid., VI, p. 613-615 pour les citations qui précèdent.

60. Ibid., VI, p. 621-623.

61. Ibid., VI, p. 623.

62. La création artistique (VS), II, p. 309.

63. La monnaie de l'absolu (VS), VI, p. 623.

64. Ibid., VII, p. 633.

65. Ibid., VII, p. 628.

66. Ibid., VII, p. 631.

67. Ibid., VII, p. 639.

68. Ibid., VII, p. 631.

\section{RÉSUMÉS}

Plus qu'à la redéfinition de l'art, c'est à la réélaboration du concept de temps requise par la découverte des œuvres préhistoriques à partir de la fin du XIX ${ }^{\mathrm{e}}$ siècle que cet article est consacré. Analysant la conception de l'histoire et du temps de deux des rares penseurs à avoir fait une place aux peintures et gravures préhistoriques dans leur histoire de l'art (E. H. Gombrich) ou leur musée imaginaire (André Malraux), il montre comment une pensée linéaire, continue et téléologique de l'histoire fait obstacle à l'intégration des productions paléolithiques dans l'histoire universelle. Au-delà d'une réflexion épistémologique sur les catégories temporelles de l'histoire de l'art, c'est de notre façon de comprendre le temps dont il est question.

\section{INDEX}

Personnes citées : Malraux (André), Gombrich (Ernst)

Mots-clés : histoire, histoire de l'art, imitation, préhistoire, primitif, progrès, temps 


\section{AUTEUR}

\section{AUDREY RIEBER}

Ancienne élève de l'École normale supérieure de Lyon, agrégée de philosophie, docteur en philosophie, Audrey Rieber s'intéresse principalement à l'art et à l'histoire qu'elle questionne à partir de la tradition philosophique (esthétique, philosophie et théorie de l'art, philosophie des formes symboliques, herméneutique, archéologie de Foucault, Kunstwissenschaft, Bildwissenschaft) mais aussi des concepts et méthodes de l'histoire (de l'art) : théorie des fondateurs (Vasari, Winckelmann, Burckhardt), iconologie (Panofsky), formalisme (Hildebrand, Riegl, Wölfflin), Kennerschaft, psychohistoire (Warburg), iconologie analytique (Arasse), École des Annales. Postdoctorante au sein du pôle de recherche eikones NFS Bildkritik (Bâle), elle a publié en 2012 Art, histoire et signification. Un essai d'épistémologie d'histoire de l'art autour de l'iconologie d'Erwin Panofsky (L'Harmattan, coll. Esthétiques). Elle est professeur invitée du Master « Aisthesis. Historische Kunst-und Literaturdiskurse » (KU Eichstätt, Allemagne). 